\title{
openheart Echocardiographic screening for the anomalous aortic origin of coronary arteries
}

\author{
Francesco Bianco (D) , 1,2 Massimo Colaneri, ${ }^{2}$ Valentina Bucciarelli, ${ }^{1,2}$ \\ Francesca Chiara Surace, ${ }^{2}$ Federica Valentina lezzi, ${ }^{2}$ Martina Primavera, ${ }^{1}$ \\ Annaclara Biasi, ${ }^{1}$ Giuliano Giusti, ${ }^{2}$ Emanuela Berton, ${ }^{2}$ Monica Baldoni, ${ }^{2}$ \\ Giulia Renda, ${ }^{1}$ Alessandra Baldinelli, ${ }^{2}$ Sabina Gallina, ${ }^{1}$ Marco Pozzi ${ }^{2}$
}

To cite: Bianco F, Colaneri M, Bucciarelli V, et al.

Echocardiographic screening for the anomalous aortic origin of coronary arteries. Open Heart 2021;8:e001495. doi:10.1136/ openhrt-2020-001495

Received 26 0ctober 2020 Revised 6 December 2020 Accepted 29 December 2020

Check for updates

(c) Author(s) (or their employer(s)) 2021. Re-use permitted under CC BY-NC. No commercial re-use. See rights and permissions. Published by BMJ.

${ }^{1}$ Department of Neuroscience, Imaging and Clinical Sciences, Universita degli Studi Gabriele d'Annunzio Chieti e Pescara,

Chieti, Abruzzo, Italy

${ }^{2}$ Pediatric and Congenital Cardiology and Cardiac Surgery, Azienda Ospedaliero Universitaria Ospedali Riuniti di Ancona Umberto I G M Lancisi G Salesi, Ancona, Marche, Italy

Correspondence to Dr Francesco Bianco; dr. francescobianco@gmail.com

\section{ABSTRACT}

Aims We sought to determine the diagnostic performance, clinical profiles and outcomes of anomalous aortic origin of coronary arteries (AAOCA) using a standardised echocardiographic approach in young adults and athletes.

Methods In 2015-2019, we screened 5998 outpatients (age 16 years (Q1-Q3: 11, 36)), referred for routine echocardiography, using four specific echocardiographic windows: parasternal short/long axis and apical 4/5-chambers view. Coronary CT confirmed AAOCA. For the performance analysis, 300 coronary-CT scans were available; two independent and double-blinded physicians retrospectively reviewed echocardiographic images.

Results A total of 47 AAOCA was diagnosed; the overall prevalence was $0.0078 \%$. Over 5 years, we found a significant increment of AAOCA diagnostic rate (P for trend=0.002). Syncope $(n=17 / 47)$ and palpitations $(n=6 / 47)$ were prevalent symptoms. All patients suspended sports activity at the diagnosis. Twenty-seven patients underwent surgery, while 20 underwent a conservative medical treatment. All patients are alive at a median follow-up of $3 \pm 1.6$ years; only surgical repairs restarted their activity. Our method showed better sensitivity than traditional short-axis evaluation: $93 \%$ vs $83 \%, p=0.0030$ (AUC $0.96(95 \% \mathrm{Cl} 0.92,0.99)$ and AUC $0.89(95 \% \mathrm{Cl} 0.83$, $0.95)$, respectively), with a good interobserver agreement $(95 \%, k=0.83, p<0.001)$.

Conclusions The application of a standardised echocardiographic approach for AAOCA detection led to a significantly increased rate of identified anomalies. This approach demonstrated higher sensitivity than the traditional echocardiographic assessment. Implementing this protocol in clinical practice may help improve the AAOCA diagnosis in young adults and athletes.

Trial registration number NCT04224090.

\section{INTRODUCTION}

The anomalous aortic origin of coronary arteries (AAOCA) is a rare congenital condition, accounting for abnormalities of origin, course, destination, size and number of the coronary vessels. ${ }^{1}$ The prevalence of the AAOCA is unclear; it ranges from $0.021 \%$ to

\section{Key questions}

What is already known about this subject?

- Anomalous aortic origin of coronary arteries (AAOCA) can result in sudden cardiac death, and echocardiography is usually the first imaging test used for screening unless it holds poor sensitivity.

What does this study add?

- We demonstrate that a standardised and routinely performed approach can ameliorate the sensitivity of the sonographic evaluation of the AAOCA.

How might this impact on clinical practice?

- A comprehensive study of the morphological features of AAOCA, based on four specific echocardiographic views that we adopted, can be used to increase the sensibility of this examination and the number of AAOCA diagnosed.

$5.79 \%$, depending on the imaging techniques used for the diagnosis: coronary angiography, cardiac magnetic resonance (CMR), CT or echocardiography. ${ }^{1}$ Though, autopsy-based study and case series of patients that experienced sudden cardiac death (SCD) due to AAOCA generally present higher frequency than imaging ones. ${ }^{12}$

The diagnosis and the screening of AAOCA can be difficult. ${ }^{3}$ The ECG is often normal or presents unspecific signs. ${ }^{4}$ Therefore, a high diagnostic suspicion is required to refer children and adolescents to invasive and ionising examinations (CT or angiography). ${ }^{5}$ In this context, echocardiography has always been considered the method of screening and diagnosis with the most limited accuracy. However, this examination is the first-line test recommended and prescribed by physicians when a congenital cardiac abnormality is suspected. ${ }^{16}$

To overcome these limitations, we designed, studied and implemented a specific echocardiographic 4-views-based approach 
to diagnose AAOCA. Therefore, our study aimed to assess the diagnostic performance, clinical profiles and outcomes of AAOCA using this standardised echocardiographic approach in young adults and athletes.

\section{METHODS}

\section{Study population and design}

The present study is a single-centre retrospective analysis. We tested the diagnostic performance of our echocardiographic-based approach for AAOCA detection and examined the clinical profiles and outcomes of the AAOCA diagnosed within these examinations. A comprehensive flow chart is presented in figure 1.

In 2015-2019, 5998 outpatients (16 years old (Q1-Q3: $11,36)$ ), both young adults and athletes, underwent routine echocardiography in our department due to the mandatory cardiovascular screening programme for the eligibility assessment to non-competitive or competitive sports activity.

The echocardiographic-based approach for AAOCA detection was systematically implemented in the department since January 2015. For our purposes, we considered only patients undergoing their first examination and thus excluded bicuspid aortic valves, known or newly discovered, already known structural or congenital heart disease, cardiomyopathies and all their longitudinal follow-up echocardiographic examinations.

All the suspected AAOCA ( $\mathrm{N}=57)$ were referred for a coronary-CT confirmation. For the performance analysis, 243 consecutive additional patients were also asked to undergo a coronary-CT scan. A total of 300 coronary-CT scans was achieved. Confirmed AAOCA were then followed-up for a median time of $3 \pm 1.6$ years.

In agreement with the Declaration of Helsinki, all patients enrolled gave written informed consent at the time of their evaluation, stating that data and images may be subsequently used for research purposes. For participants under 18 years old, a parent and/or the legal tutor gave informed consent.

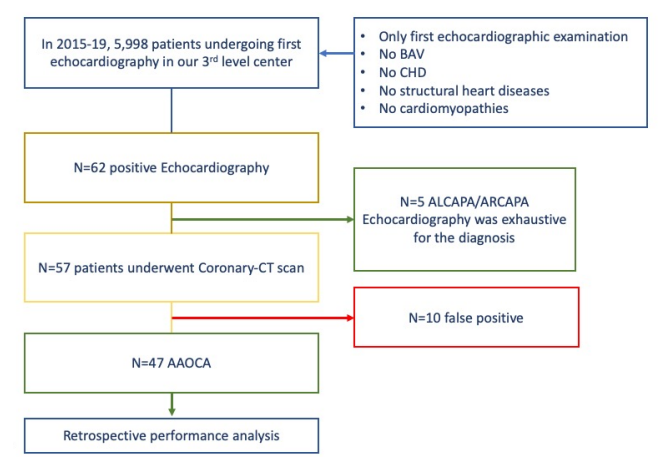

Figure 1 Study design. AAOCA, anomalous aortic origin of coronary arteries; ALCAPA, aortic left coronary artery from the pulmonary artery; ARCAPA, aortic right coronary artery from the pulmonary artery. BAV, biscuspid aortic valve; $C H D$, congenital heart disease.
Since the observational nature of the study, no ethical committee approval was required.

\section{Anomalous aortic origin of coronary arteries definition}

Coronary arteries are traditionally defined as normal when arising from the appropriate sinus of valsalva (right or left), presented a proper course (the right coronary artery (RCA) in the right atrioventricular groove; the left anterior descending (LAD) in the anterior interventricular groove, and the left circumflex (LCX) in the left atrioventricular groove) and terminated in the capillary bed. $^{78}$

For the present analysis, AAOCA were defined as anatomic variants differing from the anatomy mentioned above. In particular, the anomalous origin of the right coronary artery (AORCA) when RCA originated from the left coronary sinus of the aorta, or the non-coronary sinus, and the anomalous origin of the left coronary artery (AOLCA) when LAD or LCX originated from the right coronary sinus of the aorta or the non-coronary sinus. AAOCA, both AORCA and AOLCA, were also considered the coronary arteries arising from the aorta by a separate ostium, shared or common ostium, or as branch vessels. Besides, AAOCA were also defined as the coronary arteries that originated directly from the ascending aorta instead of valsalva's sinus. ${ }^{89}$

The aortic coronary arteries from the pulmonary artery (ACAPA) were also identified and defined as the coronary artery with an origin from the pulmonary artery: ARCAPA for the right coronary artery and ALCAPA for the left one. ${ }^{3} 10$

\section{Echocardiography}

All the echocardiographic studies were performed using a commercially available Philips iE33 ultrasound systems (Philips Medical Systems, Amsterdam, The Netherlands), using a 2.5 or $3.5 \mathrm{MHz}$ transducer, as appropriate. Images were acquired at a frame rate of 70-80 frames/s, applying a standard two-dimensional greyscale. Images of three consecutive heart cycles were collected from the parasternal short axis (PSAX) and long-axis (PLAX), and the 4/5-chambers apical views. ${ }^{10} 11$

\section{A standardised approach for the assessment of AAOCA}

Our method consisted of the visualisation of the coronary arteries from four focused-specific views: PSAX, the traditional approach, ${ }^{311}$ along with the PLAX, and the apical 4-chambers and 5-chambers view (figure 2). ${ }^{12}$ Both two-dimensional echocardiography and color-Doppler imaging were used to visualise the coronary arteries. Since the coronaries usually have low-velocity flow, the Nyquist limit was decreased to $20-40 \mathrm{~cm} / \mathrm{s}$, as recommended. ${ }^{11}$

A hallmark of AAOCA was considered the absence of a proper visualisation of the origin of the coronary arteries from the aortic root (figure 3, panels A and B) at the PSAX. ${ }^{312}$ In addition, their origin and the proximal course can be evaluated from the apical 5-chambers view, focused again on the aortic root (figure 3, panel E). 


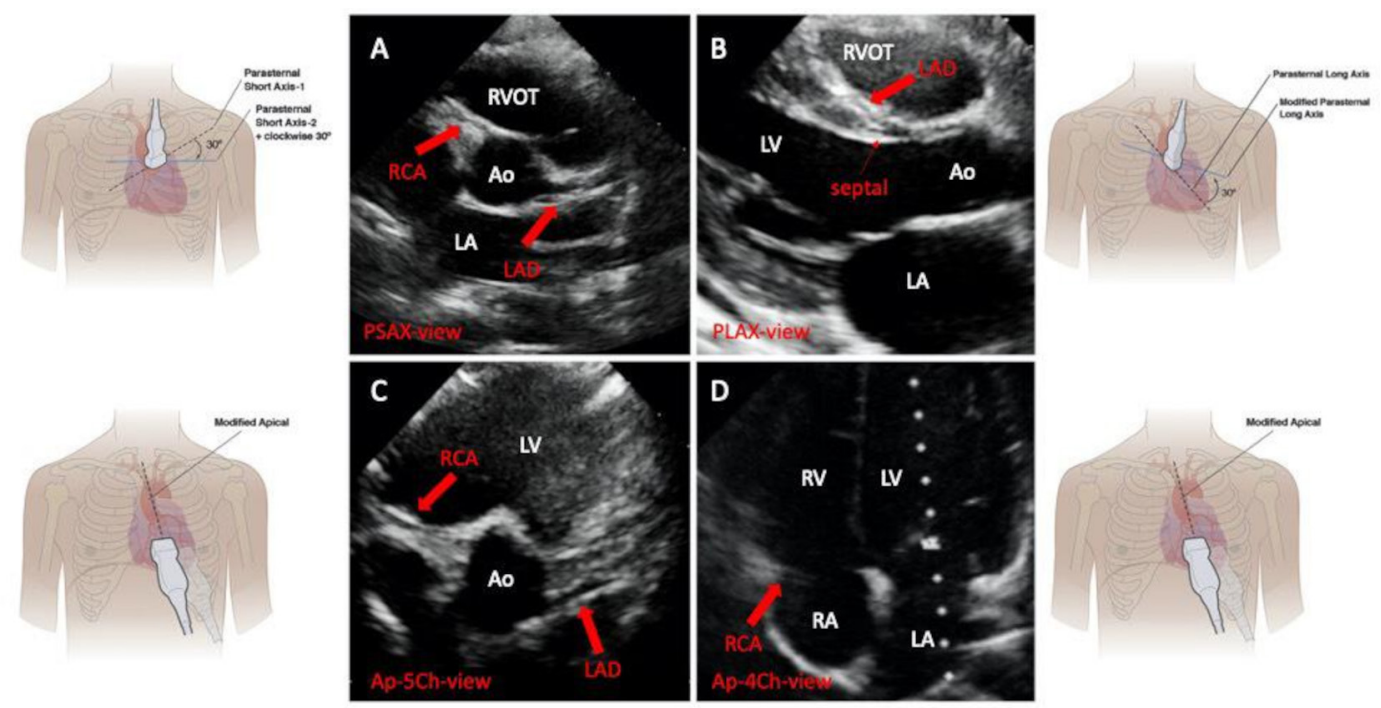

Figure 2 Two-dimensional echocardiographic 4-view approach for AAOCA detection: normal examination. Panel A: PSAX view. Panel B: PLAX view. Panel C: Ap-5Ch-view. Panel D: Ap-4Ch-view. AAOCA, anomalous aortic origin of coronary arteries; AO, aorta; Ap-4Ch-view apical four chambers view; Ap-5Ch-view, apical five chambers view; LA, left atrium; LAD, left anterior descending; LV, left ventricle; PLAX, parasternal long-axis view; PSAX, parasternal short-axis view; RA, right atrium; RCA, right coronary artery; RV, right ventricle; RVOT, right ventricular outflow tract. Modified from Lorber, R. et al. ${ }^{6} \mathrm{JACC}$ Cardiovascular Imaging 2015;8:1239-49.

We also evaluated the presence of the so-called ring sign; if present, it can be visualised from the PLAX (figure 3, panel D) and should be interpreted as atypical. ${ }^{11}{ }^{13}$ This sign is of interest when an aberrant left or right AAOCA is suspected. A coronary artery originating from the contralateral aortic sinus, along its intramural course, crosses the mid-anterior line of the circular aorta to reach the sinus of valsalva of its intended origin. Consequently, its cross-section should be visible in an orthogonal view traversing the mid-long axis of the aorta, namely, the parasternal long-axis view. ${ }^{3213}$

In normal conditions, by tilting the transducer posteriorly from the PLAX, and focusing on the RVOT, it is possible to image the left main and the left anterior descending (figure 3, panel C). An AOLCA with the LAD originating from the right sinus of valsalva should be suspected from this view in the presence of LAD and
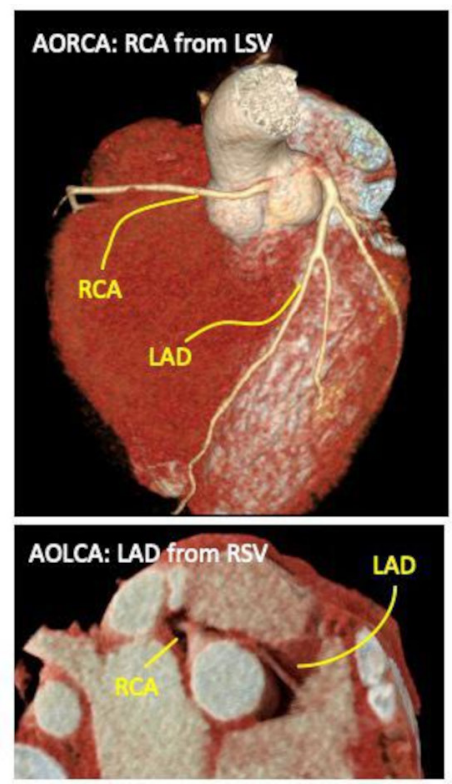
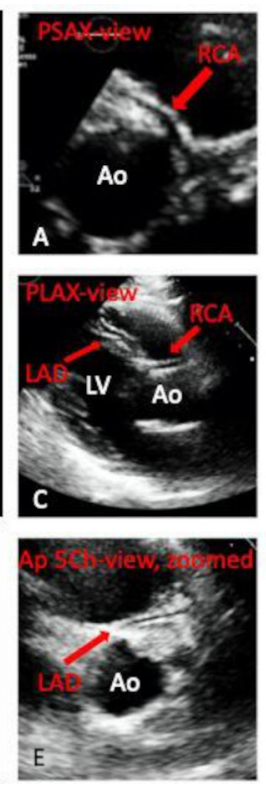
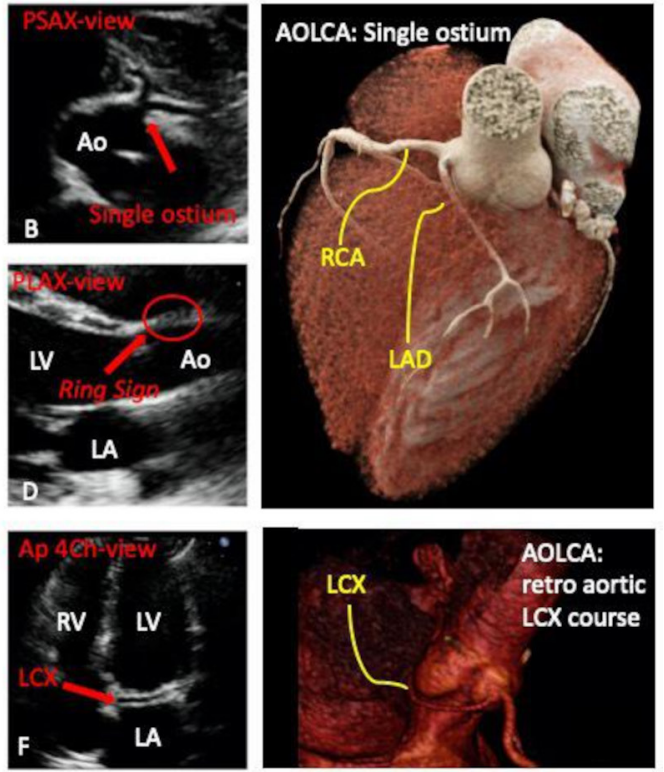

Figure 3 Two-dimensional echocardiographic 4-view approach for AAOCA detection: abnormal examinations and their anatomic correlates. AO, aorta; AOLCA, anomalous origin of the left coronary artery; AORCA, anomalous origin of the right coronary artery; Ap-4Ch-view, apical four chambers view; Ap-5Ch-view, apical five chambers view; LA, left atrium; LAD, left anterior descending; LCX, left circumflex artery; LV, left ventricle; PLAX, parasternal long-axis view; PSAX, parasternal shortaxis view; RCA, right coronary artery; RV, right ventricle; RVOT, right ventricular outflow tract. 
RCA displayed contemporarily (figure 3, panel C). ${ }^{3}$ Besides, its absence can be pathognomonic LAD agenesis or hypoplasia. ${ }^{11}$

From the apical 4-chambers view, if a coronary artery is visualised running through the left atrioventricular groove (figure 3, panel F), instead of the right atrioventricular one (figure 2, panel $\mathrm{D}$ ), a coronary retro aortic course could be suspected in both cases: LCX or LAD from the right coronary sinus. ${ }^{611}$

Usually, ACAPA are rare and do not present with a predictable pattern imaged with specific echocardiographic views. ${ }^{311}$ For ACAPA, the absence of one coronary artery from the aortic root and its appearance at the pulmonary artery level is very suggestive and used for this analysis. ${ }^{311}$

\section{Coronary CT}

All coronary-CT examinations were obtained using a 384 (192×2)-slices third-generation scanner (SOMATOM Force CT; Siemens Healthineers, Forchheim, Germany). Images were acquired at the isotropic resolution of $0.5-0.75 \mathrm{~mm}$ in a $25 \mathrm{~cm}$ field of view. Patients received a non-ionic lowosmolality contrast agent (iodixanol; GE Healthcare Life Sciences, Chalfont, UK), with a volume of $30-120 \mathrm{~mL}$ $(1-1.5 \mathrm{~mL} / \mathrm{kg})$ and flow injection rate of $3-5 \mathrm{~mL} / \mathrm{s}$, followed by saline injection administered intravenously using a power injector at same volume and velocity. ${ }^{5} \mathrm{~A}$ bolus triggering was used to scan initiation. The scans were acquired with a $70-120 \mathrm{kV}$ of tube voltage with an automatic tube current modulation technique (CAREdose). A retrospective ECG-triggered axial coronary protocol was applied. $^{5}$

\section{Performance analysis}

According to our internal policy, echocardiographic and CT images are stored and available in our Institutes' Core Lab for further analysis. Two independent physicians (MP and ACB), both experts in echocardiography and AAOCA, retrospectively reviewed the 5998 echocardiographic images and re-examined them in a double-blinded fashion from the coronary-CT examinations and diagnosis, ignoring the aim of this re-examination. For the performance analysis, 300 coronary-CT scans were available and were considered as the gold standard.

Separately for each patient, the physicians reviewed both the short-axis view, focused on the aortic root (twodimensional images only and images with colour, the traditional approach for AAOCA detection) ${ }^{1611}$ and our proposed method (based on the visualisation of four standardised echocardiographic views); then, were asked to decide if the coronary anatomy can be considered as usual or not. The physicians can access the full echocardiographic examination, except the specific 4-views, while examining the traditional approach. A QLab software (Philips Medical Systems, Amsterdam, The Netherlands) was used for the offline retrospective analysis.

\section{Statistical analysis}

Categorical variables are expressed as percentages, while continuous variables as mean and $\mathrm{SD}( \pm \mathrm{SD})$ or median and IQR $(\mathrm{Q} 1, \mathrm{Q} 3)$, as appropriate. The prevalence was derived by comparing the number of people found to have an AAOCA with the total number of people studied. The incidence was estimated as the prevalence per year.

Statistical comparisons between continuous variables (the number of AAOCA, misdiagnosis and types of AAOCA) across the 5-year period of investigation were made with the non-parametric trend test. Continuous variables were compared by the Student's t-test or non-parametric, while categorical variables were compared by the $\chi^{2}$ test or Fisher's exact test, when appropriate. Receiver operating curve characteristics were used to assess the sensibility and specificity of each method (the traditional PSAX and the proposed 4-views approach) and compared through regression analysis.

The interobserver agreement for the adjudicated doubleblinded retrospective diagnosis was assessed by means of the kappa (k) statistic coefficient. A k of 1 indicated perfect agreement, a $\mathrm{k}$ of 0 indicates poor agreement, the associated significance of the $p$ value indicated that the estimated $\mathrm{k}$ was not caused by chance alone.

A two-tailed $p$ value of 0.05 was considered statistically significant. All statistical analyses were performed with Stata V.14.1 (Stata Corp, College Station, Texas, USA) and Prism V.8.0 (GraphPad Software, La Jolla, California, USA).

\section{RESULTS \\ General characteristics}

In our cohort, coronary abnormalities were suspected in 62 patients using echocardiography. Of them, five were diagnosed as ARCAPA or ALCAPA; the echocardiograms were considered as exhaustive, without the need for further investigations. The latter underwent surgery for coronary repair. Besides, 57 patients underwent a coronary-CT scan for the diagnosis confirmation of AAOCA. These examinations discovered 10 false positives, while a total of 47 AAOCA were confirmed.

The overall prevalence of AAOCA was $0.0078 \%$, with $0.0015 \%$ of incidence. The $22.8 \%(\mathrm{n}=13 / 47)$ AAOLCA $)$, while the $59.6 \%(\mathrm{n}=34 / 47)$ were AAORCA. The RCA from the left coronary sinus with an inter-arterial course was the most frequent AAOCA 56.1\% (N=37). Regarding AAOLCA, the LCX from the right coronary sinus with a retro-aortic course $(8.8 \%, \mathrm{~N}=5)$ was the most frequent.

\section{Echocardiographic screening results}

After the implementation in 2015 of our echocardiographicbased method of diagnosis, we found a progressive increment of the number of the AAOCA identified ( $P$ for trend=0.002), growing progressively through the years 2015-2019 (table 1). A rise not accompanied by an increased number of misdiagnosis ( $\mathrm{P}$ for trend $=0.20$ ). Of note, we observed that our method determined a significant progression of the AAOCA diagnosis: both AOLCA 
Table 1 Annual diagnostic rate of coronary arteries abnormalities, from 2015 to 2019

\begin{tabular}{|c|c|c|c|c|c|c|c|}
\hline & Total & 2015 & 2016 & 2017 & 2018 & 2019 & \\
\hline & $(\mathrm{N}=5998)$ & $(\mathrm{N}=1065)$ & $(\mathrm{N}=1165)$ & $(\mathrm{N}=1171)$ & $(\mathrm{N}=1562)$ & $(\mathrm{N}=1035)$ & $P$ for trend \\
\hline \multicolumn{8}{|l|}{ Diagnosis } \\
\hline AAOCA, n (\%) & $47(0.8 \%)$ & $3(0.3 \%)$ & $6(0.5 \%)$ & $10(0.9 \%)$ & $12(0.8 \%)$ & $16(1.5 \%)$ & 0.002 \\
\hline Misdiagnosis, n (\%) & $10(0.2 \%)$ & $3(0.3 \%)$ & $2(0.2 \%)$ & $2(0.2 \%)$ & $3(0.3 \%)$ & $0(0.0 \%)$ & 0.20 \\
\hline \multicolumn{8}{|l|}{ Types of AAOCA } \\
\hline AOLCA, n (\%) & $13(0.2 \%)$ & $0(0.0 \%)$ & $1(0.1 \%)$ & $3(0.3 \%)$ & $3(0.2 \%)$ & $6(0.6 \%)$ & 0.007 \\
\hline AORCA, n (\%) & 34 (0.6 \%) & $3(0.3 \%)$ & $5(0.4 \%)$ & 7 (0.6 \%) & $9(0.6 \%)$ & 10 (1.0 \%) & 0.042 \\
\hline
\end{tabular}

Data are categorical and described as counts (proportions).

AAOCA, anomalous aortic origin of coronary arteries; AOLCA, anomalous origin of the left coronary artery; AORCA, anomalous origin of the right coronary artery.

and AORCA ( $\mathrm{P}$ for trend=0.007 and $\mathrm{P}$ for trend 0.042, respectively).

We acknowledged 10 misdiagnoses of the total 57 suspected AAOCA that underwent the coronary-CT scan for confirmation. Most of them $(\mathrm{N}=5 / 10)$ was due to the para-commissural coronary position to the aortic valve $(\mathrm{p}=0.029)$. The overlap with nearby structure at the echocardiography and the presence of a separate conal ramus were also found to be secondary causes of echocardiographic images misinterpretation.

\section{Clinical profiles and outcomes}

In our cohort of AAOCA, the male sex was prevalent $(85.1 \%, \mathrm{n}=40 / 47)$, while $65.9 \%(\mathrm{n}=31)$ of participants

Table 2 Clinical profiles and demographic characteristics of AAOCA diagnosed in 2015-2019

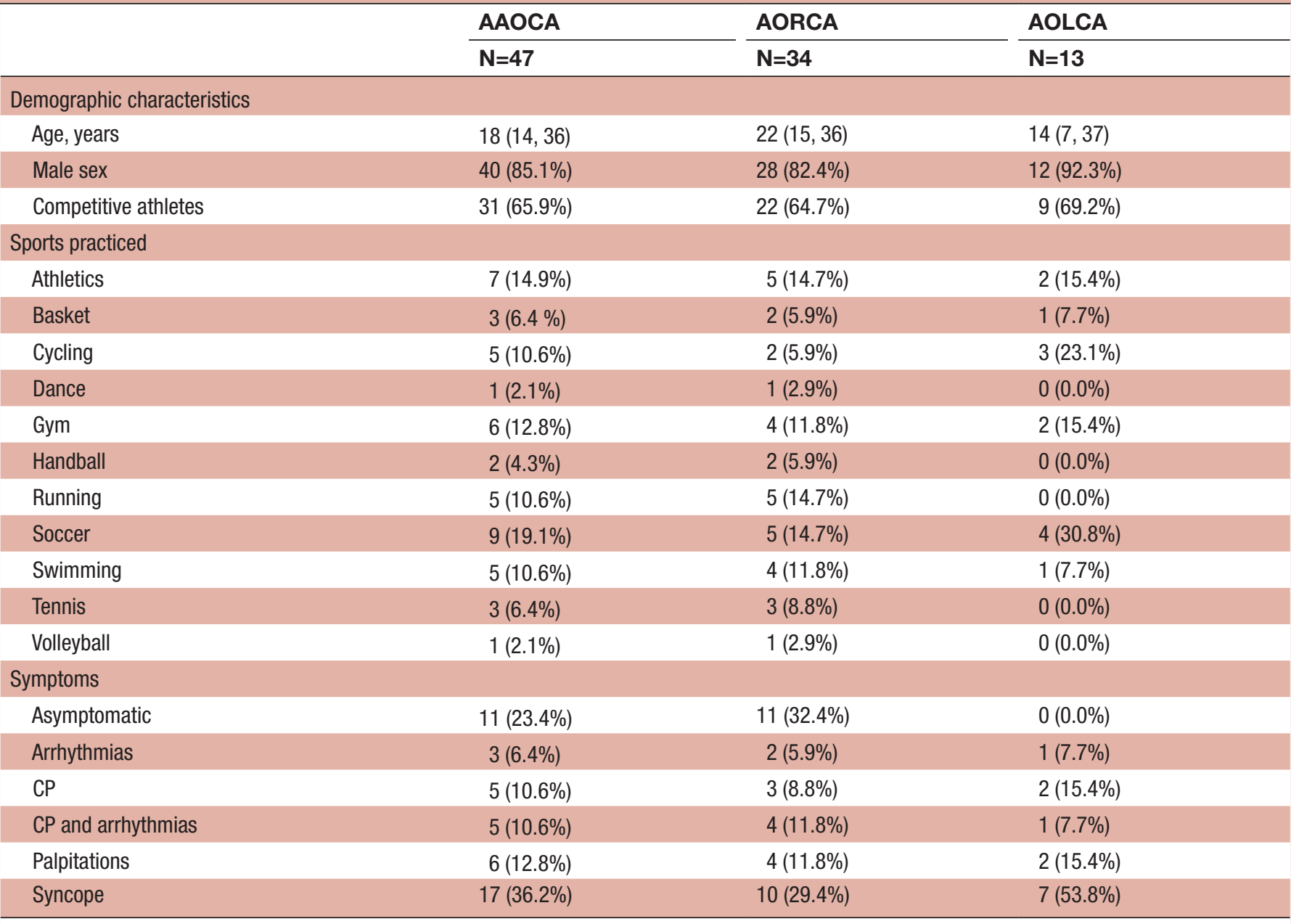

AAOCA, anomalous aortic origin of coronary arteries; AOLCA, anomalous origin of the left coronary artery; AORCA, anomalous origin of the right coronary artery; $\mathrm{CP}$, chest pain. 
Table 3 Diagnostic performance of PSAX-view and four-views echocardiographic diagnostic approach for AAOCA

\begin{tabular}{|c|c|c|c|c|c|c|c|c|}
\hline Echocardiographic methods & AUC & $95 \% \mathrm{Cl}$ & Sensitivity & Specificity & LR+ & LR- & PPV & NPV \\
\hline PSAX-view approach & 0.89 & $(0.83,0.95)$ & $83 \%$ & $96 \%$ & 5 & 0.17 & 79.5 & 96.6 \\
\hline Four-views approach & 0.96 & $(0.92,0.99)$ & $93 \%$ & $99 \%$ & 20 & 0.06 & 95.6 & 98.7 \\
\hline
\end{tabular}

Results of receiver operating characteristic (ROC) curves analysis.

AAOCA, anomalous aortic origin of coronary arteries; AUC, area under the curve; LR, likelihood ratio; NPV, negative predictive value; PPV, positive predictive value.

practised competitive sports (table 2). AORCA were older than AOLCA (22 years old vs 14 years old). The majority of AORCA was asymptomatic $(\mathrm{n}=11,32.4 \%)$. Syncope $(\mathrm{n}=17 / 47)$ and palpitations $(\mathrm{n}=6 / 47)$ were prevalent symptoms in both AORCA and AOLCA.

All patients suspended sports activity at the diagnosis. Twenty-seven patients underwent surgical repair when surgery was applicable due to high-risk anatomy, ${ }^{14}$ while 20 underwent conservative medical treatment, following currently available guidelines. ${ }^{15}$ Regarding the surgery techniques, the majority underwent coronary unroofing $(n=22 / 27)$; three patients experienced coronary reimplantation, one patient a lateral coronary translocation and one the Vouhé's osteoplasty.

To the best of our knowledge, no SCD were reported among the 5998 outpatients that we screened. Regarding the AAOCA that we detected, all patients are alive at a median follow-up of $3 \pm 1.6$ years. Sport restriction was more frequent in AOLCA than AORCA: 8 (23\%) vs 5 (38\%), $\mathrm{p}<0.001$ ( $\chi^{2}$ test). Only patients that underwent surgical repair restarted the sports activity. Besides, a participant developed ischaemic heart disease and underwent percutaneous coronary stenting of the LCX (AAOCA: LCX retro-aortic curse). The latter was not operated because not currently indicated from guidelines. ${ }^{14}$

\section{Diagnostic performance analysis}

The detailed results are shown in table 3. Compared with the traditional echocardiographic approach for the AAOCA detection, centred on the PSAX coronary evaluation and color-Doppler evaluation, the 4-views approach, that we adopted, determined a significant increment of the echocardiographic sensitivity in our examinations: $93 \%$ vs $83 \%, \mathrm{p}=0.0030$ (AUC $0.96,95 \%$ CI $0.92,0.99$ and AUC 0.89, 95\% CI 0.83, 0.95, respectively). When adding the adjunctive echocardiographic views, the positive predictive value of the echocardiographic evaluation augmented from 79.5 up to 96.6, resulting in more conclusive than traditional (positive likelihood ratio $>20$ and negative $<0.1$ ).

Finally, we found a good interobserver agreement between physicians for the adjudicated double-blinded retrospective diagnosis of AAOCA (95\%, $\mathrm{k}=0.83$, $\mathrm{p}<0.001)$.

\section{DISCUSSION}

In the present study, whereas implementing our echocardiographic-based approach for coronary abnormalities detection, we observed a progressive and statistically significant increment in AAOCA diagnosed through 2015-2019. This increment determined an augmentation of the AAOCA identified with the echocardiography. In this cohort, AOLCA were younger and more symptomatic than AORCA; syncope was prevalent, while, after the AAOCA diagnosis, only patients that underwent surgical repair restarted sports activity. No significant events were recorded during a median follow-up of $3 \pm 1.6$ years. Our method showed better sensitivity than the traditional PSAX evaluation of the origin and proximal course of the coronary arteries, with an excellent interobserver agreement between physicians at the retrospective analysis.

Traditionally, echocardiography is considered the imaging technique with the most limited accuracy for the coronary arteries evaluation. ${ }^{1}$ Despite this belief, echocardiography is the first-line imaging examination that physicians recommend in young patients and athletes when cardiac abnormalities are suspected. ${ }^{16}$ The echocardiographic assessment of AAOCA is generally done from the PSAX, focused on the aortic root, trying to visualise the origin and the proximal course of the coronary arteries. ${ }^{1}$ Other acoustic windows are possible, along with a color-Doppler evaluation and probe tilts to off-axis views, ${ }^{11}$ but they are less implemented in everyday practice, excluding specialised centres in paediatric cardiology and congenital heart diseases. ${ }^{6}$ The present study adds to previous literature by demonstrating that a standardised and routinely performed approach can ameliorate the sensitivity of the sonographic evaluation of the AAOCA.

The approach that we tested, except the PLAX, focused on the RVOT, is generally part of the routine echocardiographic evaluations. ${ }^{310}$ While the 4 -views approach we proposed can be regularly acquired, both by physicians and sonographers. Since this method does not require advanced skills but only little practice (in our experience, no more than 2 or 3 weeks), it can be systematically implemented; these images can be stored, re-examined or sent to experts for a second look, at any time, without altering or interfering with their sensitivity and specificity. Besides, even if in young patients it is infrequent to find poor acoustic windows, it can easily overcome this limitation due to its multiview approach.

Since AAOCA can be associated with SCD, particularly during efforts, ${ }^{4}$ the diagnosis should be pursued in particular populations and contexts. ${ }^{289}$ According 
to the previous literature, ${ }^{4}$ we found that AOLCA were generally younger and more symptomatic than AORCA; a history of syncope is frequent, while chest pain and palpitations are less common. On the contrary, AORCA are often asymptomatic, and, accordingly, the age of the diagnosis is usually higher.

Some authors deny the association mentioned above, ${ }^{17}$ while others consider this condition as a possible predisposition to accelerated atherosclerosis, ${ }^{18}$ but autopsybased series generally report a relatively more significant number of SCD occurred in young patients and athletes with AAOCA, both AORCA and AOLCA. ${ }^{2} 1920$ Consequently, implementing this simple echocardiographic 4-views approach in everyday practice, as we demonstrated, could increase the number of diagnoses within a second level examination, even in subjects asymptomatic, and therefore reduce the amount of some AAOCArelated SCD during sports activities.

\section{Limitations and strengths}

One of the principal limitations of this study is that it comprises only a single-centre experience in the AAOCA echocardiographic evaluation. Our analysis's second limitation is that we do not know the exact amount of the overall false negative, but only 300 cases. By the way, we assume that this number has been little. According to our policy, we usually prescribe a second examination within 8-12 months from the first evaluation, even if the first echocardiogram is considered negative for cardiac abnormalities. So, these double-check between operators and evaluations can reduce misdiagnoses. We believe that some missed AAOCA would have been reported during these years in which we implemented our protocol, but, to date, no missed AAOCA have been reported at these second examinations.

Moreover, other centres across the world usually adopt multiviews and color-Doppler approaches for the AAOCA detection; so, our study suffers from a lack of novelty in this view, but these approaches are not encoded, studied and standardised yet.

\section{Clinical perspectives}

AAOCA echocardiographic diagnosis is challenging, and imaging protocols have not been standardised yet. Unlike other approaches, ${ }^{1611}$ we opted for a standardised method and thus reduced the need for peculiar individual skills and expertise.

This protocol can be easily implemented in sonographic laboratories; the images can be stored and available for further evaluations, especially in the view of telemedicine support, if the examinations are carried out in peripheric centres or sports medicine laboratories. Besides, the method we proposed is not time-consuming since it involves standard echocardiographic windows.

\section{CONCLUSIONS}

The application of a new echocardiographic-based method for AAOCA detection led to a significantly increased rate of identified anomalies and AAOLCA. This approach demonstrated better sensitivity than the traditional echocardiographic assessment, with excellent reliability among physicians. Implementing this protocol in clinical practice may reduce AAOCA-related sudden cardiac death in young adults and athletes.

Acknowledgements Special thanks to all the staff of the Department of Pediatric and Congenital Cardiology and Cardiac Surgery - Azienda Ospedaliero Universitaria "Ospedali Riuniti" - Ancona, Italy.

Collaborators Alessandro Capestro, Elli Soura, Ettore Merlino, Gaetano Santoro.

Contributors $\mathrm{FB}$ and $\mathrm{MC}$ designed the study and drafted the manuscript. $\mathrm{FB}$ performed the statistical analysis. VB, FCS and FV revised the manuscript and contributed to the analysis and interpretation of the data. ACB and MP contributed to the retrospective analysis. $E B, G G, M B$ and $A B$ contributed to the clinical evaluation and support to the recruitment of patients. $G R$ and $A B$ were involved in critical revision and data interpretation. SG and MP revised the final manuscript and supported the study design.

Funding This study was conducted with the support of Regione Marche, Italy (grant reference: 4/SPV 15/02/2019).

Competing interests None declared.

Patient consent for publication Obtained.

Provenance and peer review Not commissioned; externally peer reviewed.

Data availability statement All data relevant to the study are included in the article or uploaded as supplementary information.

Open access This is an open access article distributed in accordance with the Creative Commons Attribution Non Commercial (CC BY-NC 4.0) license, which permits others to distribute, remix, adapt, build upon this work non-commercially, and license their derivative works on different terms, provided the original work is properly cited, appropriate credit is given, any changes made indicated, and the use is non-commercial. See: http://creativecommons.org/licenses/by-nc/4.0/.

ORCID iD

Francesco Bianco http://orcid.org/0000-0001-9785-3402

\section{REFERENCES}

1 Cheezum MK, Liberthson RR, Shah NR, et al. Anomalous Aortic Origin of a Coronary Artery From the Inappropriate Sinus of Valsalva. J Am Coll Cardiol 2017;69:1592-608.

2 Frescura C, Basso C, Thiene G, et al. Anomalous origin of coronary arteries and risk of sudden death: a study based on an autopsy population of congenital heart disease. Hum Pathol 1998;29:689-95.

3 Brown LM, Duffy CE, Mitchell C, et al. A practical guide to pediatric coronary artery imaging with echocardiography. J Am Soc Echocardiogr 2015;28:379-91.

4 Finocchiaro G, Behr ER, Tanzarella G, et al. Anomalous Coronary Artery Origin and Sudden Cardiac Death: Clinical and Pathological Insights From a National Pathology Registry. JACC Clin Electrophysiol 2019;5:516-22.

5 Saremi F. Cardiac CT and Mr for adult congenital heart disease. New York: Springer, 2014.

6 Lorber R, Srivastava S, Wilder TJ, et al. Anomalous aortic origin of coronary arteries in the young: echocardiographic evaluation with surgical correlation. JACC Cardiovasc Imaging 2015;8:1239-49.

7 Angelini P. Normal and anomalous coronary arteries in humans. coronary artery anomalies: a comprehensive approach. Philadelphia: Lippincott Williams \& Wilkins, 1999.

8 Villa AD, Sammut E, Nair A, et al. Coronary artery anomalies overview: the normal and the abnormal. World J Radiol 2016;8:537-55.

9 Pérez-Pomares JM, de la Pompa JL, Franco D, et al. Congenital coronary artery anomalies: a bridge from embryology to anatomy and pathophysiology--a position statement of the development, anatomy, and pathology ESC Working Group. Cardiovasc Res 2016;109:204-16.

10 Mitchell C, Rahko PS, Blauwet LA, et al. Guidelines for performing a comprehensive transthoracic echocardiographic examination in adults: recommendations from the American Society of echocardiography. J Am Soc Echocardiogr 2019;32:1-64. 
11 Frommelt P, Lopez L, Dimas VV, et al. Recommendations for multimodality assessment of congenital coronary anomalies: a guide from the American Society of echocardiography: developed in collaboration with the Society for cardiovascular angiography and interventions, Japanese Society of echocardiography, and Society for cardiovascular magnetic resonance. J Am Soc Echocardiogr 2020;33:259-94

12 Stoebe S, Lange K, Pfeiffer D, et al. Feasibility of proximal right coronary artery imaging by $2 \mathrm{D}$ and $3 \mathrm{D}$ echocardiography in comparison to coronary angiography. Echo Res Pract 2015;2:73-9.

13 Jureidini SB, Marino CJ, Singh GK, et al. Aberrant coronary arteries: a reliable echocardiographic screening method. J Am Soc Echocardiogr 2003;16:756-63.

14 Ong CS, Cameron DE, Jacobs ML. Surgical management of anomalous coronary arteries. Ann Cardiothorac Surg 2018;7:604-10.

15 Stout KK, Daniels CJ, Aboulhosn JA, et al. 2018 AHA/ACC guideline for the management of adults with congenital heart disease: a report of the American College of Cardiology/American heart association Task force on clinical practice guidelines. Circulation 2019;139:e698-800.

16 Malhotra A, Dhutia H, Finocchiaro G, et al. Outcomes of cardiac screening in adolescent soccer players. N Engl J Med 2018;379:524-34.

17 Silverman KJ, Bulkley BH, Hutchins GM. Anomalous left circumflex coronary artery: "normal" variant of uncertain clinical and pathologic significance. Am J Cardiol 1978;41:1311-4.

18 Roberts WC, Waller BF, Roberts CS. Fatal atherosclerotic narrowing of the right main coronary artery: origin of the left anterior descending or left circumflex coronary artery from the right (the true "left-main equivalent"). Am Heart J 1982;104:638-41.

19 Basso C, Maron BJ, Corrado D, et al. Clinical profile of congenital coronary artery anomalies with origin from the wrong aortic sinus leading to sudden death in young competitive athletes. J Am Coll Cardiol 2000;35:1493-501.

20 Eckart RE, Campbell CL, Shry EA. Annals of internal medicine article sudden death in young adults: a 25-year review of autopsies in military recruits. Ann Intern Med 2004;141:829-35. 